\title{
CONDIÇÕES DE PRODUÇÃO VOCAL DE VENDEDORES DE MÓVEIS E ELETRODOMÉSTICOS: CORRELAÇÃO ENTRE QUESTÕES DE SAÚDE, HÁBITOS E SINTOMAS VOCAIS
}

\author{
Vocal production conditions for furniture and household \\ appliance salespeople: correlation between health issues \\ and vocal symptoms and habits
}

Léslie Piccolotto Ferreira ${ }^{(1)}$, Patrícia Luciano ${ }^{(2)}$, Clara Megumi Akutsu ${ }^{(3)}$

\section{RESUMO}

Objetivo: analisar as condições de produção vocal, considerando saúde geral, hábitos vocais, assim como seus sintomas e possíveis causas, em vendedores de móveis e eletrodomésticos. Métodos: foram selecionados 100 vendedores de móveis e eletrodomésticos, de ambos os sexos, em seu próprio local de trabalho, para responderem 16 questões que pesquisou dados pessoais, saúde geral, hábitos e sintomas vocais. Os dados foram analisados com auxílio do programa Statistical Package for Social Sciences, em sua versão 13.0. Resultados: 66 homens e 34 mulheres, com idade média de 24 anos e tempo médio de profissão de 8,5 anos apontaram em maior número os distúrbios de saúde geral, relacionados às questões emocionais $(31 \%)$, alterações no sono $(27 \%)$ e problemas digestivos (25\%); as alterações auditivas, como coceira no ouvido (36\%), intolerância a sons altos (29\%), e dificuldade para ouvir (20\%). Quanto aos hábitos relacionados à voz, fizeram referência a falar muito $(83 \%)$, tomar gelado $(73 \%)$ e tomar café $(68 \%)$. Os sintomas mais apontados foram garganta e boca seca $(30 \%)$, cansaço ao falar $(22 \%)$ e pigarro (18\%). As possíveis causas para a ocorrência dos sintomas foram uso intenso da voz (49\%), presença de poeira $(27 \%)$ e de ar condicionado (23\%). Apenas quatro deles relataram ter alteração de voz. Conclusão: os vendedores pesquisados percebem a presença dos sintomas vocais, mas não os relacionam com alterações de voz, assim como desconhecem os cuidados com a mesma. Necessitam, portanto, de ações de promoção de saúde e prevenção de alterações vocais, com o objetivo de sensibilizá-los com relação a essas questões.

DESCRITORES: Voz; Perfil de Saúde; Distúrbios da Voz

(1) Fonoaudióloga; Professora Titular do Departamento de Fundamentos da Fonoaudiologia da Pontifícia Universidade Católica de São Paulo (Professora da Faculdade de Fonoaudiologia e do Programa de Estudos Pós-Graduados em Fonoaudiologia); Coordenadora e Docente do Curso de Especialização em Fonoaudiologia - Voz - Pontifícia Universidade Católica de São Paulo/Coordenadoria Geral de Especialização, Aperfeiçoamento e Extensão; Doutora em Distúrbios da Comunicação Humana pela Universidade Federal de São Paulo.

(2) Fonoaudióloga; Clínica de Fonoaudiologia Comunicavoz; Bacharel em Fonoaudiologia pela Pontifícia Universidade Católica de São Paulo.

(3) Fonoaudióloga; Clínica de Fonoaudiologia Comunicavoz; Bacharel em Fonoaudiologia pela Pontifícia Universidade Católica de São Paulo.

Pesquisa de Iniciação Cientifica realizada com bolsa PIBIC/ CNPq, no Curso de Fonoaudiologia da Pontifícia Universidade Católica de São Paulo. Brasil.

\section{INTRODUÇÃO}

A Fonoaudiologia nos últimos anos, ao discutir os aspectos da voz profissional, tem analisado as possíveis causas de alterações vocais decorrentes do trabalho como conseqüência de fatores do próprio organismo dos sujeitos ou advindas de fatores ambientais e organizacionais. Dessa forma, depois de diagnosticar alterações vocais, parte para o tratamento das mesmas e para as ações de promoção para prevenção de novas alterações vocais, na tentativa de minimizar problemas vocais e melhorar a performance do trabalhador que atua com a voz profissionalmente ${ }^{1}$.

Dentre esses profissionais, pode-se destacar ao lado de professores, teleoperadores, radialistas, 
atores, entre outros, os vendedores. Esses são profissionais que utilizam a voz como meio de trabaIho e de maneira continuada, os quais procuram, por meio de um modo de expressão própria atingir um público específico e determinado ${ }^{2}$. Num sentido amplo, o vendedor pode ser definido como um indivíduo que age como o intermediário entre o proprietário dos bens e a pessoa que deseja adquirir esses bens ${ }^{3}$. O uso da voz no contexto da comercialização existe há muito tempo e na literatura são encontrados relatos das diferentes formas e produtos que são comercializados, sendo esses anunciados muitas vezes unicamente por meio da voz.

Atualmente é possível observar que houve um aumento de lojas de móveis e eletrodomésticos, e a concorrência, com as chamadas de grandes ofertas, faz com que aumente o trabalho e a carga horária dos vendedores, que trabalham em média nove horas por dia fazendo uso da voz, para tentar conquistar a atenção do cliente, que é seduzido, por meio desta, além dos recursos corporais ${ }^{4}$.

$\mathrm{O}$ vendedor assume um compromisso com a empresa de vender o produto, prestar assistência ao cliente, criar uma imagem positiva da empresa e distinguir os clientes que irão efetivar a compra dos que estão apenas observando ${ }^{3}$.

$\mathrm{Na}$ observação do contexto de trabalho nesse ramo de atuação (móveis e eletrodomésticos), constata-se que geralmente as lojas estão localizadas em avenidas de grande circulação de ônibus, carros e transeuntes. Os vendedores muitas vezes se posicionam na porta do estabelecimento, à espera de um cliente e, em alguns casos, gritam para chamar a sua atenção, para que entrem na loja.

No momento da venda, os vendedores podem utilizar recursos e técnicas da construção de um determinado personagem para enfatizar a mercadoria. Muitas vezes, esses profissionais abusam da voz por falarem incessantemente com a utilização do ar de reserva na tentativa de convencer o cliente. Nesse momento, o produto é mostrado com entusiasmo, pois estes profissionais têm a necessidade de envolver o cliente, transmitir confiança e sinceridade para conquistá-lo e alcançar o objetivo de vender ${ }^{4}$.

Visto que este é um ambiente de trabalho, no qual o vendedor fala demasiadamente e algumas vezes grita, certas vezes, ele ultrapassa os limites saudáveis para a sua voz, quer seja por desconhecimento das normas básicas da produção vocal, quer seja por imitação de um modo vocal inadequado ${ }^{5}$.

Em decorrência de um abuso vocal excessivo, o vendedor pode apresentar um distúrbio vocal e, assim, a voz não cumprir o seu papel profissional. Esse impedimento da produção natural da voz pode aparecer como sintoma principal ou sintoma secundário a uma outra doença, fato que gera diversos transtornos na vida do profissional que tem a voz como instrumento de trabalho ${ }^{6}$. Esse comportamento abusivo da voz também pode causar cansaço e esforço vocal, o que produz na seqüência, vozes roucas e tensas que interferem na boa comunicação ${ }^{6}$.

Existem relatos de distúrbios de voz relacionados ao trabalho que decorrem mais dos fatores ambientais e organizacionais do que do próprio trabalho e é preciso distinguir as vozes prejudicadas pelo ambiente profissional (gases, calor, ruído, etc) das vozes alteradas pelo uso abusivo ou inadequado.

O foco para possíveis causas sobre o uso abusivo da voz pode estar na presença de distúrbios alérgicos, faríngeos, bucais, nasais, otológicos, pulmonares, digestivos, hormonais, neurovegetativos e medicamentoso ${ }^{6}$.

Os problemas vocais podem também estar associados a componentes emocionais, pois a voz expressa as emoções ${ }^{6}$. Assim, questões da esfera psíquica desempenham um papel importante no desenvolvimento de alguns distúrbios vocais, uma vez que a voz revela o estado emocional. Por este motivo, algumas pessoas submetidas a situações de stress, angústia ou estado de euforia podem sentir mudanças na altura tonal, intensidade ou entoação de sua voz e podem inclusive ficar sem voz por algum período de tempo ${ }^{6}$.

A escassez de pesquisas, relacionadas a vendedores que utilizam a voz como instrumento de trabaIho justifica a realização deste estudo, que tem por objetivo analisar as condições de produção vocal considerando saúde geral, hábitos vocais, assim como sintomas vocais e suas possíveis causas.

\section{MÉTODOS}

Participaram desta pesquisa de caráter descritivo e prospectivo, 100 vendedores de lojas de móveis e eletrodomésticos, de ambos os sexos, contatados por conveniência em seu próprio local de trabalho.

Foi elaborado, a partir do questionário proposto pelo Comitê de Voz e Telemarketing da Sociedade Brasileira de Fonoaudiologia, um instrumento para coleta de dados, em versão resumida e adaptada para vendedores. Depois de submetê-lo a dois vendedores que participaram do piloto desta pesquisa, o instrumento foi ajustado (Figura 1), e totalizou ao final 16 questões que procuraram levantar dados pessoais (1 a 11), saúde geral (12 e 13), hábitos vocais (14), sintomas vocais (15) e possível causa desses sintomas (16). 


\section{AVALIAÇÃO OCUPACIONAL DA VOZ FALADA PARA VENDEDORES}

Caro Vendedor, você foi escolhido(a) para responder o questionário a seguir. É muito importante que você responda corretamente a todas as questões, pois certamente você estará contribuindo para a melhoria da sua profissão. Contamos com a sua colaboração!!!

Para as questões de número 7, 8 e 9, além de marcar o que se sente, é preciso também marcar a freqüência. Portanto, marque:

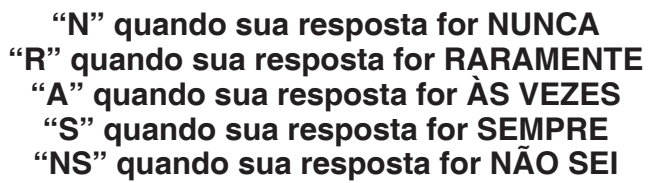

NÚMERO:

\section{DADOS PESSOAIS}

1. Data:

2. Tempo de profissão:

3. Data de nascimento: ..................

$\begin{array}{lll}\text { 4. Sexo: 1. ( ) masculino 2. ( ) feminino } & \text { 2.... }\end{array}$

5. Carga horária semanal:

6. Você tem ou já teve alteração na sua voz?
0. ( ) não
1. ( ) sim, tive. Qual?
2. ( ) sim, tenho. Qual?

7. II. SAÚDE GERAL
7.1 ( ) hormonal
0. ( ) N 1.( )R 2. ( ) A 3. ( ) S 4. ( ) NS
7.2 ( ) digestivo
0. ( ) $N$ 1. ( )R 2. ( ) A 3. ( ) S 4. ( ) NS
7.3 ( ) emocional
0. () N 1. () R 2. () A 3. () S 4. () NS
7.4 ( ) coluna
7.5 ( ) circulatório
0. ( ) N 1. ( )R 2.( ) A 3. ( ) S 4. ( ) NS
7.6 ( ) dificuldade com o sono
0. ( ) N 1. ( ) R 2. ( ) A 3. ( ) S 4. ( ) NS
7.7 ( ) rinite
7.8 ( ) sinusite
0. ( ) $\mathrm{N}$ 1.( ) R 2.( ) A 3.( ) S 4.( ) NS
0. ( ) N 1.( )R 2. ( ) A 3. ( ) S 4. ( ) NS
0. ( ) $\mathrm{N}$ 1.( ) R 2.( ) A 3.( ) S 4.( ) NS

8. Saúde Auditiva: (marque uma ou mais alternativas)
8.1 ( ) dificuldade de ouvir
0. ( ) N 1.( ) R 2.( ) A 3. ( ) S 4. ( ) NS
8.2 ( ) coceira no ouvido
0. ( ) $N$ 1.( ) R 2. ( ) A 3. ( ) S 4. ( ) NS
8.3 ( ) intolerância a sons altos
0. ( ) N 1.( ) R 2.( ) A 3. ( ) S 4.( ) NS

9. Hábitos relacionados à voz, imediatamente anterior e/ou durante o período de trabalho:
9.1 ( ) toma gelado
0. ( ) N 1. ( ) R 2. ( ) A 3. ( ) S 4. ( ) NS
9.2 ( ) toma café
0. ( ) N 1.( ) R 2.( ) A 3.( ) S 4.( ) NS
9.3 ( ) ingere leite, queijos
0. ( ) $\mathrm{N}$ 1.( ) R 2.( ) A 3.( ) S 4.( ) NS
9.4 ( ) come chocolate
0. ( ) $N$ 1.( ) R 2.( ) A 3.( ) S 4.( ) NS
9.5 ( ) fala muito
0. ( ) $N$ 1. ( ) R 2. ( ) A 3. ( ) S 4. ( ) NS
9.6 ( ) fala muito
0. ( ) N 1.( ) R 2.( ) A 3.( ) S 4.( ) NS

10. Sintomas vocais presentes nas 2 últimas semanas: (marque uma ou mais alternativas)

10.1 ( ) cansaço ao falar

10.2 ( ) ardor na garganta / dor ao falar

10.3 ( ) falhas na voz

10.4 ( ) pigarro

10.5 ( ) garganta/boca seca

10.6 ( ) voz pior pela manhã

11. Na sua opinião, o(s) sintoma(s) assinalados na pergunta anterior acontece $(\mathrm{m})$ porque: (marque uma ou mais alternativas)

11.1 ( ) uso intenso da voz

11.2 ( ) presença de ar condicionado

11.3 ( ) presença de poeira

11.4 ( ) presença de ar condicionado

11.5 ( ) estresse relacionado ao trabalho

Figura 1 - Protocolo utilizado na avaliação 
A maioria das questões foi elaborada com o padrão de respostas em escalas de freqüência ( 1 - raramente, 2 - às vezes, 3 - sempre), e algumas com espaço para detalhamento das respostas (exemplo: existe estresse no ambiente de trabalho? Sim/não; se há, especifique o motivo).

A aplicação do protocolo foi feita pela própria pesquisadora diretamente com o vendedor no seu local de trabalho, em média durante 15 minutos. Para não comprometer as respostas, apenas depois de concluído cada vendedor assinou o Termo de Consentimento.

A pesquisa foi aprovada pelo Comitê de Ética da Pontifícia Universidade Católica de São Paulo, sob o n 0239/06.

Para evitar possíveis erros os dados foram duplamente digitados em planilha específica, e submetidos à análise estatística descritiva.

\section{RESULTADOS}

A população estudada foi composta por 66 homens e 34 mulheres, com idade média de 24 anos, com tempo médio de profissão de 8,5 anos e com uma carga horária média de 54 horas/semanais.

Os vendedores apontaram em maior número, como distúrbios de saúde geral, aqueles relacionados a questões emocionais $31(31 \%)$, rinite 28 $(28 \%)$, alterações do sono 27 (27\%), problemas digestivos $25(25 \%)$, problemas de coluna $23(23 \%)$, sinusite 17 (17\%), alterações circulatórias 14 (14\%) e hormonais 10 (10\%) (Tabela 1).

Tabela 1 - Distribuição percentual (\%) quanto à presença ou ausência de distúrbios de saúde geral mencionado pelos vendedores $(n=100)$

\begin{tabular}{lcc}
\hline \multirow{2}{*}{ Variável } & Presença & Ausência \\
\cline { 2 - 3 } & $\%$ & $\%$ \\
\hline Emocional & 31 & 69 \\
Rinite & 28 & 72 \\
Sono & 27 & 73 \\
Digestivo & 25 & 75 \\
Coluna & 23 & 77 \\
Sinusite & 17 & 83 \\
Hormonal & 10 & 90 \\
Bronquite & 6 & 94 \\
Psiquiátrico & 5 & 95 \\
Amigdalite & 5 & 95 \\
Circulatório & 4 & 86 \\
Neurológico & 3 & 97 \\
Asma & 2 & 98 \\
Faringite & 2 & 98 \\
Outros* & 2 & 98 \\
Laringite & 0 & 100 \\
\hline Os & 2 &
\end{tabular}

Os outros sintomas apresentados pelos vendedores
Dentre as queixas relacionadas às alterações auditivas, os vendedores fizeram referência em maior número à coceira no ouvido $36(36 \%)$, intolerância a sons altos 29 (29\%), e dificuldade para ouvir 20 (20\%) (Tabela 2).

Tabela 2 - Distribuição percentual (\%) quanto à presença ou ausência de distúrbios de saúde auditiva mencionada pelos vendedores $(n=100)$

\begin{tabular}{lcc}
\hline \multirow{2}{*}{ Variável } & Presença & Ausência \\
\cline { 2 - 3 } & $\%$ & $\%$ \\
\hline Coceira no Ouvido & 36 & 64 \\
Intolerância a Sons Altos & 29 & 71 \\
Dificuldade de Ouvir & 20 & 80 \\
Zumbido & 13 & 87 \\
Dor de Ouvido & 12 & 88 \\
\hline
\end{tabular}

Quanto aos hábitos relacionados à voz, os vendedores afirmaram que falam muito $83(83 \%)$, tomam gelado $73(73 \%)$, tomam café $68(68 \%)$, ingerem derivados de leite $61(61 \%)$, comem chocolate 56 (56\%), gritam 27 (27\%) e fumam 24 (24\%) (Tabela 3).

Tabela 3 - Distribuição percentual (\%) quanto à presença ou ausência dos hábitos relacionados à voz mencionados pelos vendedores $(n=100)$

\begin{tabular}{lcc}
\hline \multirow{2}{*}{ Variável } & Presença & Ausência \\
\cline { 2 - 3 } & $\%$ & $\%$ \\
\hline Fala muito & 83 & 17 \\
Ingere bebida gelada & 73 & 27 \\
Ingere café & 68 & 32 \\
Ingere derivados do leite & 61 & 39 \\
Ingere chocolate & 56 & 44 \\
Grita & 27 & 73 \\
Fuma & 24 & 76 \\
\hline
\end{tabular}

Parte dos vendedores apontou ter algum sintoma vocal e os mais citados foram garganta e boca seca $30(30 \%)$, cansaço ao falar $22(22 \%)$, voz pior pela manhã $19(19 \%)$, pigarro $18(18 \%)$, ardor na garganta $11(11 \%)$ e falha na voz $11(11 \%)$ (Tabela 4).

As possíveis causas para a ocorrência dos sintomas vocais, na opinião dos vendedores foram: uso intenso da voz 49 (49\%), presença de poeira $27(27 \%)$ e de ar condicionado 23 (23\%). Quando questionados sobre os motivos de stress relacionado ao trabalho, os vendedores relataram que a causa está relacionada ao fato de terem um "horá- 
Tabela 4 - Distribuição percentual (\%) quanto à presença ou ausência de sintomas vocais mencionados pelos vendedores $(n=100)$

\begin{tabular}{lcc}
\hline \multirow{2}{*}{ Variável } & Presença & Ausência \\
\cline { 2 - 3 } & $\%$ & $\%$ \\
\hline Garganta/boca seca & 30 & 70 \\
Cansaço ao falar & 22 & 78 \\
Piora pela manhã & 20 & 80 \\
Pigarro & 18 & 92 \\
Voz grossa & 18 & 82 \\
Ardor na garganta & 11 & 89 \\
Falhas na voz & 11 & 89 \\
Voz fraca & 11 & 89 \\
Esforço ao falar & 9 & 91 \\
Perda de voz & 7 & 93 \\
Voz forte & 6 & 94 \\
Piora pela noite & 6 & 94 \\
Voz fina & 2 & 98 \\
\hline
\end{tabular}

Tabela 5 - Distribuição percentual (\%) quanto à presença ou ausência de possíveis causas de alterações de vocais mencionados pelos vendedores $(n=100)$

\begin{tabular}{lcc}
\hline \multirow{2}{*}{ Variável } & Presença & Ausência \\
\cline { 2 - 3 } & $\%$ & $\%$ \\
\hline Uso vocal intenso & 49 & 51 \\
Stress & 35 & 65 \\
Presença de poeira & 27 & 73 \\
$\begin{array}{l}\text { Presença de ar } \\
\text { condicionado }\end{array}$ & 23 & 77 \\
Mudança brusca de & 21 & 79 \\
temperatura & & \\
Ambiente & & \\
excessivamente & 20 & 80 \\
frio ou quente & & \\
Ruído no ambiente & 18 & 82 \\
Presença de fumaça & 17 & 83 \\
Escassez de intervalo & 16 & 84 \\
Motivo & 14 & 86 \\
Uso de produto de & 13 & 87 \\
limpeza & & \\
Relacionamento & 3 & 97 \\
insatisfatório & 1 & 99 \\
Presença de carpete & 1 & 99 \\
Mobiliário inadequado & & \\
\hline
\end{tabular}

rio puxado", falarem muito, cobrança e pressão para vender, cansaço, falta de bem estar entre os funcionários e por não receberem um valor justo quanto à comissão por horas extras trabalhadas (Tabela 5).

Apenas quatro vendedores, todos do sexo feminino, responderam ter no presente ou no passado alguma alteração de voz.

\section{DISCUSSÃO}

Nesta pesquisa, sem a pretensão inicial de igualar a amostra quanto à variável sexo, encontrou-se mais homens do que mulheres na função de vendedor. Essa não é a mesma realidade encontrada entre professores ou entre teleoperadores em que mais mulheres ocupam os postos de trabalho ${ }^{7,8}$. Por outro lado, se a literatura faz referência ao sexo feminino ser mais predisposto a distúrbios vocais, o fato de mais homens participarem desta pesquisa também pode explicar o número menor de sujeitos que fizeram referência a distúrbio de voz quando comparada a outras pesquisas realizadas com professores ${ }^{7,9}$. Tal fato é ainda reforçado pelo número de vendedores que disseram ter no presente ou no passado alteração de voz. Apesar de ser um número reduzido, como foi comentado anteriormente, todos são do sexo feminino.

A população estudada foi composta em média por adultos jovens, considerando a idade e o tempo de trabalho. Este é outro fator que pode explicar a pouca ocorrência de distúrbios vocais nessa população, pois em idade mais avançada é mais comum ocorrerem distúrbios de voz ${ }^{10}$. Pode se considerar que o período de máxima eficiência vocal ocorre entre 25 e 45 anos, sendo que a partir dessa idade uma série de alterações estruturais na laringe, com maior ou menor impacto, pode ser identificada.

Os sujeitos pesquisados atuam em carga horária semanal superior à duração regulamentada para o trabalho de empregados em qualquer atividade privada (oito horas diárias) segundo a Consolidação das Leis do Trabalho (CLT) vigente no país. Tal fato aponta para a possibilidade do cumprimento de horas extras por parte desses vendedores, com o alerta que esse excesso pode interferir nas questões de saúde dos trabalhadores (www.trt02.gov.br/ geral/tribunal2/legis/CLT).

As manifestações nas vias respiratórias (asma, rinite e sinusite) quando presentes predispõem à presença de distúrbios vocais, principalmente quando o sujeito passa a exercer uma profissão em que haja o uso mais efetivo da voz. Essas afecções de vias respiratórias costumam também se manifestar na presença de variação brusca de temperatura e presença do ar condicionado ${ }^{11}$, fatores mencionados pelos pesquisados.

Sobre o ambiente de trabalho, os vendedores, assim como feirantes, professores e teleoperadores ${ }^{7,8,12}$ afirmam trabalhar em lugar ruidoso, com temperatura elevada ou reduzida demais, o que demanda um maior esforço para concentração de atenção. $O$ ruído intenso no ambiente de trabaIho exige ainda que se eleve a voz para a comunicação, o que pode ocasionar alterações vocais 
importantes ${ }^{7,12,13}$. As outras conseqüências do ruído na saúde podem resultar em alteração do sono, irritabilidade, problemas gástricos, disfunções hormonais ${ }^{14}$, entre outras questões que também foram mencionadas pelos vendedores.

Quanto aos hábitos relacionados à voz, os vendedores evidenciaram desconhecer os possíveis cuidados com a voz, um dos principais instrumentos de seu dia-a-dia profissional ${ }^{10}$. Especificamente com relação ao tabagismo, mais de um quinto dos vendedores fez referência ao hábito de fumar. Essa porcentagem é diferente dos achados na literatura: é encontrada em maior número, em pesquisa realizada, com acadêmicos de Medicina da cidade de Sorocaba ${ }^{15}$, em idade próxima dos sujeitos desta pesquisa (17,17\%); e é inferior aos dados da pesquisa realizada com funcionários de estabelecimentos educacionais do Chile, com média de idade superior dos participantes desta pesquisa (40 anos $40,7 \%{ }^{16}$. Apesar de um estudo ${ }^{17}$ revelar que o consumo de tabaco tem diminuído entre os adultos jovens, acredita-se que por esse hábito ser um dos principais dentre aqueles que interferem na qualidade vocal ${ }^{18}$, atenção especial deve ser dada nas ações de promoção de saúde e de prevenção de alterações vocais.

Os hábitos apresentados pelos vendedores podem ser classificados como inadequados, pois uma má alimentação, falar muito e tomar gelado são fatores que impedem um bom desempenho vocal e esses são profissionais que não possuem, em sua formação, informações necessárias para um bom cuidado com a voz. Isso também acontece com professores e teleoperadores, segundo dados de pesquisas realizadas com esses profissionais ${ }^{7,19,20}$.

De acordo com os dados levantados nesta pesquisa, o vendedor é um profissional da voz que apresenta sintomas relacionados a distúrbios vocais, embora não refira ter no presente ou passado uma alteração vocal na mesma proporção que professores, teleoperadores, feirantes, e outros profissionais que também utilizam a voz como instrumento de trabalho $8,12,21$.

Além dos aspectos apresentados (o fato de o maior número de participantes ser do sexo masculino e em idade jovem), um dos possíveis fatores do vendedor não fazer referência a alteração vocal, é que ele não necessita usar a voz em intensidade mais elevada como o professor. $O$ vendedor tem auxílio, além do uso da voz, de gestos e do apoio visual (aspectos que o teleoperador não dispõe), pois no momento da compra, se o cliente não gostar de determinado produto, existem outras opções, fato que faz com que o vendedor não faça tanto esforço vocal para convencer o cliente, como no caso do teleoperador que utiliza somente sua voz e fala para poder cativar o cliente e atingir sua meta ${ }^{8}$.

Dessa forma, a atuação fonoaudiológica de caráter preventivo é fundamental, para qualquer profissional que faça uso da voz, especificamente para o vendedor, pois nas últimas décadas, houve um aumento na preocupação com a voz profissional e com as questões dos fatores ambientais e organizacionais de trabalho, que interferem na produção vocal ${ }^{6}$. O alerta de que a prática fonoaudiológica deve considerar o dia-a-dia do profissional para que possa ser efetiva, enfatizado junto à atuação com professores ${ }^{22}$, deve ser considerado também no trabalho com os vendedores.

Certamente iniciativas do fonoaudiólogo, nessa direção, da mesma forma como tem sido feitas com teleoperadores ${ }^{23}$, auxiliarão o vendedor, que necessita cumprir com sua produtividade e que faz uso da sua voz, seja no trabalho e/ou em suas relações sociais.

\section{CONCLUSÃO}

Os vendedores pesquisados fazem menção a uma série de distúrbios de saúde geral e hábitos vocais que podem comprometer a produção da voz. Percebem a presença de sintomas vocais, mas não os relacionam a alterações de voz. Necessitam, portanto de ações de promoção de saúde e prevenção de alterações vocal, com o objetivo de sensibilizá-los com relação a essas questões.

\section{AGRADECIMENTOS}

Agradecimentos especiais aos integrantes do Laboratório de Voz - Laborvox - da PUC-SP, e ao CNPq, pela bolsa que viabilizou a realização desta pesquisa. 


\begin{abstract}
Purpose: to analyze the vocal production conditions for furniture and household appliance salespeople, considering the aspects of general health, vocal habits, as well as vocal symptoms and their possible causes. Methods: 100 furniture and household appliance salespeople of both genders were selected in their own work environment, to answer a questionnaire composed of 16 questions concerning personal information, issues on general health, vocal habits and symptoms. Data were digitized and analyzed using SPSS (Statistical Package for Social Sciences) version 13.0. Results: the sample was composed of 66 men and 34 women, with an average age of 24 years of age, working in this occupation for an mean time of 8.5 years. The subjects mostly reported general health disorders, related to emotional $(31 \%)$, sleep (27\%), and digestive $(25 \%)$ issues; in auditory disorders, such as ear-itch (36\%), intolerance to loud sounds (29\%), and hearing difficulty $(20 \%)$. As far as vocal habits, the aspects mentioned were excessive talking (83\%), fluid intake in cold temperatures $(73 \%)$ and drinking coffee $(68 \%)$. The symptoms that were most reported were dry throat and mouth $(30 \%)$, vocal fatigue $(22 \%)$ and phlegm (18\%). The possible causes associated to these symptoms by the participants in the study were intense vocal usage (49\%), presence of dust $(27 \%)$ and air-conditioning $(23 \%)$ in work environment. Only four subjects reported having a voice disorder. Conclusion: the salespeople at issue note the occurrence of vocal symptoms, but not those related to voice disorders. They are also unaware of vocal care habits. Therefore, they need health promotion and vocal disorder prevention actions, aiming at making them aware on these health issues.
\end{abstract}

KEYWORDS: Voice; Health Profile; Voice Disorders

\section{REFERÊNCIAS}

1. Ferreira LP. Assessoria fonoaudiológica aos profissionais da voz. In: Ferreira LP, Befi-lopes DM, Limongi SCO. Tratado de fonoaudiologia. São Paulo: Roca; 2004.

2. Sataloff RT. Professional voice the science and art of clinical care. New York: Raven Press; 1991.

3. Soldow GF, Thomas GP. Vendas: profissionalização para a década de 1990. São Paulo: Makron Books; 1993.

4. Caldo LV. A voz do vendedor como meio de "sedução" na venda. [monografia] São Paulo: Pontifícia Universidade Católica de São Paulo; 2001.

5. Behlau MS. Vozes preferidas: considerações sobre opções vocais nas profissões. Fono Atual. 2001; 4(16):10-4.

6. Centro de Vigilância em Saúde. Distúrbios de voz relacionados ao trabalho. Bol Epidemiol Paulista. 2006; 26(3):16-23.

7. Ferreira LP, Giannini SPP, Figueira S, Silva EE, Karmann DF e Souza TMT. Condições de produção vocal de professores da prefeitura do município de São Paulo. Rev Dist Comun. 2003; 14(2):275-308.

8. Algodoal MJAO. Voz profissional: o operador de telemarketing. [dissertação]. São Paulo (SP): Pontifícia Universidade Católica de São Paulo; 1995.
9. Ferreira LP, Benedetti PH. Condições de produção vocal de professores de deficientes auditivos. Rev CEFAC. 2007; 9(1):79-89.

10. Boone DR. Inimigos biológicos da voz profissional. Pró-Fono. 1992; 4(2):3-8.

11. Minitti A, Bento RF, Butugan O. Otorrinolaringologia: clínica e cirúrgica. São Paulo: Atheneu; 1993.

12. Silvério KCA, Mourão LF, Fedosse E, Trevisor TT, Granato C. Comparação do uso vocal de feirantes nas cidades de Piracicaba e São Paulo. Rev Dist Comun. 2003; 15(1):39-58.

13. Libardi A, Gonçalves CGO, Vieira TPG, Silvério KCA, Rossi D, Penteado RZ. O ruído em sala de aula e a percepção dos professores de uma escola de ensino fundamental de Piracicaba. Rev Dist Comun. 2006; 18(2):167-78.

14. Roy N, Merril RM, Gray SD, Smith EM. Voice disorders in the general population: prevalence, risk factors, and occupational impact. Laryngoscope. 2005; 115(11):1988-95.

15. Rosemberg J, Perón S. Tabagismo entre estudantes da faculdade de ciências médicas de Sorocaba: tabagismo nos acadêmicos de medicina e nos médicos. J Pneumol. 1990; 16(1):13-22.

16. Bello SS, Soto MI, Michalland SH, Salinas JC. Encuesta nacional de tabaquismo en funcionarios de salud. Rev Méd Chile. 2004; 132(2):223-32.

17. Spiegel JR, Sataloff RT, Emerich KA. The young adult voice. J Voice. 1997; 11(2):138-43. 
18. Figueiredo DC, Souza PRF, Gonçalves MIR, Biase NG. Análise perceptivo-auditiva, acústica computadorizada e laringológica da voz de adultos jovens fumantes e não-fumantes. Rev Bras Otorrinolaringol. 2003; 69(6):791-800.

19. Jones K, Sigmon J, Hock L, Nelson E, Sullivan $\mathrm{M}$, Ogren F. Prevalence and risk factors for voice problems among telemarketers. Arch Otolaryngol Head Neck Surg. 2002; 128(5):571-7.

20. Fletcher HM, Drinnan MJ, Carding PN. Voice care knowledge among clinicians and people with healthy voices or dysphonia. J Voice. 2007; 21(1):80-91.
21. Medeiros AM, Barreto SM, Assunção AA. Voice disorders (dysphonia) in public school female teachers working in Belo Horizonte: prevalence and associated factors. J Voice. 2008; 22(6):676-87.

22. Penteado ERZ. Relações entre saúde e trabalho docente: percepções de professores sobre saúde vocal. Rev Soc Bras Fonoaudiol. 2007; 12(1):18-22.

23. Lehto L, Alku P, Bäckström T, Vilkman E. Voice symptoms of call-centre customer service advisers experienced during a work-day and effects of a short vocal training course. Logop Phon Vocol. 2005; 30(1):14-27.

RECEBIDO EM: 30/10/2007

ACEITO EM: 05/06/2008

Endereço para correspondência:

Rua Domingos Veja, 776

São Paulo - SP

CEP: 02837-000

Tel: (11) 3921-8168

E-mail: patricia.luciano2007@ ig.com.br 